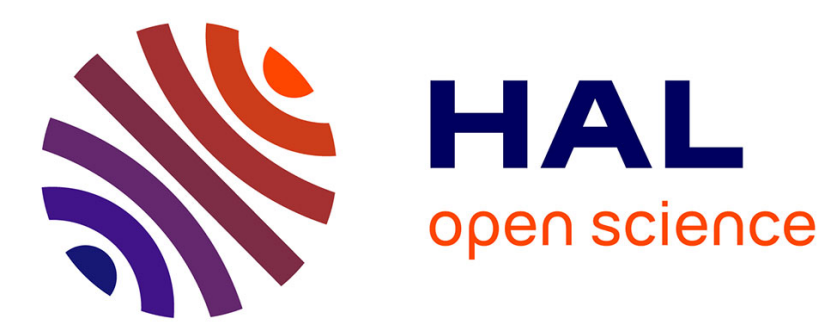

\title{
Comparaison de différentes méthodes de détermination de l'âge individuel chez le vison (Mustela vison, Schreiber)
}

\author{
M. Pascal, P. Delattre
}

\section{- To cite this version:}

M. Pascal, P. Delattre. Comparaison de différentes méthodes de détermination de l'âge individuel chez le vison (Mustela vison, Schreiber). Canadian Journal of Zoology, 1981, 59 (2), pp.202-211. 10.1139/z81-034 . hal-02197021

\section{HAL Id: hal-02197021 \\ https://hal.science/hal-02197021}

Submitted on 29 Jul 2019

HAL is a multi-disciplinary open access archive for the deposit and dissemination of scientific research documents, whether they are published or not. The documents may come from teaching and research institutions in France or abroad, or from public or private research centers.
L'archive ouverte pluridisciplinaire HAL, est destinée au dépôt et à la diffusion de documents scientifiques de niveau recherche, publiés ou non, émanant des établissements d'enseignement et de recherche français ou étrangers, des laboratoires publics ou privés. 


\title{
Comparaison de différentes méthodes de détermination de l'âge individuel chez le vison (Mustela vison Schreiber)
}

\author{
M. PASCAL \\ Laboratoire de la Faune Sauvage et Cynégétique, Centre National de la Recherche Zootechnique, \\ 78350 Jouy en Josas, France
}

et

Equipe de Recherche "Formations squelettiques," Laboratoire d'Anatomie comparée, Université Paris 7, 2, Place Jussieu, 75221 Paris, France

ET

\begin{abstract}
P. Delattre
Laboratoire de la Faune Sauvage et Cynégétique, Centre National de la Recherche Zootechnique, 78350 Jouy en Josas, France
\end{abstract}

Reçu le 3 juin 1980

Pascal, M., et Delatrre P. 1981. Comparaison de différentes méthodes de détermination de l'âge individuel chez le vison (Mustela vison Schreiber). Can. J. Zool. 59: 202-211.

Un lot de visons (322) d'âge connu provenant d'élevages ont servi à établir la relation entre le poids des cristallins et l'âge. Parmi ces individus, 27 ont servi à l'étude histologique de l'os mandibulaire, de la diaphyse fémorale, du cément et de la dentine de la M1 et de la canine. Le poids des cristallins constitue un bon critère d'âge jusquà 1 an et demi; chez les animaux plus âgés c'est le dénombrement des lignes d'arrêt de croissance (L.A.C.) du cément acellulaire de la région du collet de la M1 qui constitue le meilleur critère d'âge individuel. L'époque due dépôt des L.A.C. est précisée.

Pascal, M., and Delattre P. 1981. Comparaison de différentes méthodes de détermination de l'age individuel chez le vison (Mustela vison Schreiber). Can. J. Zool. 59: 202-211.

The relationship between lens weights and age was determined using 322 farm minks of known age. Twenty-seven of these animals were used in the histological study of the mandibular bone, the femoral diaphysis, the cementum and dentine of $M 1$ and the canine tooth. The weight of the lenses represents a good age indicator until 1.5 years. In older animals counting the resting or adhesive line (L.A.C., ligne d'arrêt de croissance) of the acellular cementum in the region of the MI constitutes the best criterion for determining the individual age. The period of L.A.C. deposition is indicated more accurately.

\section{Introduction}

Pour établir rapidement et en toute sécurité la validité d'un critère d'âge il est indispensable de l'éprouver sur un lot relativement important d'animaux d'âge connu. Nous avons profité de l'occasion qui nous a été offerte d'étudier un lot de visons (Mustela vison) d'âge connu en provenance de l'élevage expérimental de l'Institut National de la Recherche Agronomique pour comparer plusieurs techniques de détermination de l'âge individuel et rechercher la plus performante, la plus rapide ainsi que les limites de chacune d'elles.

Les études portant sur les critères d'âge chez le Vison ont surtout développé l'analyse de données morphométriques ou pondérales, appliquées à différents organes ou pièces squelettiques. C'est ainsi qu'Elder (1951) a étudié l'évolution du poids, de la taille et de la morphologie du baculum chez des animaux d'âge connu et est parvenu, grâce à ces critères, à séparer les juvéniles des adultes. Dans un même ordre d'idée, Lechleitner (1954) a analysé des donnés portant sur le poids de la carcasse dépouillée, la longueur totale du corps, le poids du baculum et sa morphologie, l'aspect et la longueur de fémur et 20 paramètres crâniométriques. Cet auteur parvient ainsi à un résultat identique: quel que soit le critère utilisé, les juvéniles peuvent être distingués des adultes, mais aucune ségrégation ne peut être opérée avec une certaine sécurité dans ce dernier groupe. Birney et Fleharty (1966) aboutissent à une conclusion similaire après avoir comparé et étudié 47 paramètres morphométriques ou pondéraux portant sur différentes pièces squelettiques. Aulerich et Swindler (1968) ont analysé la chronologie de l'apparition des dents chez le Vison et en donne un calendrier permettant d'attribuer un âge très précis entre la naissance et 3 mois. Passé cet âge, ce critère devient caduc. L'étude de l'usure dentaire sur le lot d'animaux dont nous disposons nous a conduit à la conclusion qu'il n'existe aucune corrélation précise entre l'âge individuel et l'usure dentaire.

Quelques travaux traitant de la structure et de la dynamique des populations de visons ont été réalisés en utilisant de tels critères morphométriques (Hibbard 1956; Paul 1968). Cependant, ces applications restent limitées, dans la mesure où seules deux classes d'âge sont prises en considération. C'est pour tenter de pallier 
cet inconvénient que nous avons voulu développer deux techniques peu ou pas employées, à notre connaissance, sur les Mustélidés à partir d'animaux d'âge connu. Il s'agit, d'une part, de l'évolution du poids des cristallins en fonction de l'âge et, d'autre, part, de la méthode squelettochronologique, c'est-à-dire la détermination de l'âge individuel par l'interprétation des structures histologiques des os ou des dents (Castanet et al. 1977).

La pesée des cristallins a été abondamment employée pour déterminer l'âge chez les Rongeurs et les Lagomorphes. On trouvera une bibliographie détaillée dans l'article de synthèse de Bourlière et Spitz (1975).

L'étude des marques squelettiques de croissance a elle aussi déjà fourni de nombreux résultats positifs dans différents groupes de Mammifères (voir Klevezal et Kleinenberg (1967), Sergeant (1967), Jonsgard (1969), Morris (1970), Bourlière et Spitz (1975), en particulier, chez les carnivores (Stonberg et Jonkel 1966; Jensen et Nielsen 1968; Craighead 1970; Grue et Jensen 1973; Stephenson 1977; inter alia). Cette technique consiste à dénombrer les couches d'apposition successives du tissu osseux, dentaire ou péridentaire. En effet, de larges "zones" homogènes sont séparées les unes des autres soit par des lignes fines appelées "lignes d'arrêt de croissance" (L.A.C.) (Castanet 1974) soit par des structures plus épaisses: les annuli. S'il est possible de démontrer pour un taxon particulier que le mode de dépôt de ces zones, de ces L.A.C. ou de ces annuli est un phénomène cyclique de périodicité constante et connue, leur simple dénombrement renseigne sur l'âge de l'animal.

Chez les Mustélidés, Klevezal et Kleinenberg (1967) ont étudié la Zibeline (Martes zibelina), la Loutre marine (Enhydra lutris) et le Vison. Ces auteurs ont trouvé dans la mandibule de ces différentes espèces une bonne corrélation entre le nombre de L.A.C. et l'âge des animaux. Chez le Vison, ils précisent cependant que les lignes surnuméraires peuvent perturber les déterminations de l'âge et entraîner une certaine confusion. Toujours chez le Vison, ils notent la présence de lignes hématoxylinophiles dans le cément circumradiculaire de la canine, sans préciser cependant s'il existe une corrélation entre le nombre de ces marques et l'âge des animaux. Van Soest et Van Bree (1970) ont trouvé une bonne convergence entre différents critères d'âge et le nombre de L.A.C. dans le cément de la canine de Mustela erminea. Ils n'ont cependant pu disposer d'animaux d'âge connu pour confirmer leurs résultats. Sur le Blaireau (Meles meles), Ahnlund (1976) a obtenu une bonne convergence entre le nombre de L.A.C. dans la dentine de la canine et d'autres critères d'âge. Dans ce cas aussi l'absence de collection de référence n'a pas permis d'établir avec certitude ce résultat.

Le plus souvent, donc, les auteurs ont fixé leur atten- tion sur un seul type de tissu calcifié soit l'os, soit le cément, soit la dentine. Nous avons voulu les étudier simultanément afin d'établir si ces trois tissus donnent des résultats concordants et corrélés avec l'âge individuel et si tel n'est pas le cas, de déterminer lequel de ces tissus constitue le meilleur enregistreur pratique de l'âge chez le Vison.

\section{Matériel et méthodologie}

\section{Les animaux}

Les animaux sontélevés dans des cages individuelles situées en plein air et sont par conséquent soumis aux conditions variables de température et d'éclairement de la région parisienne. Ils sont nourris quotidiennement, ad libitum, de pâtée à base de poisson.

Les 321 individus (166 mâles, 155 femelles) mis à notre disposition appartiennent à deux souches, l'une de couleur pastel, l'autre de couleur noire (tableau 1). Les sujets se répartissent en six classes d'âge, de 6 mois à 5 ans et demi. La période de mise bas se situant en mai-juin et le sacrifice des animaux au mois de décembre, l'écart entre chaque classe est exactement de 1 an.

\section{Le poids des cristallins}

Nous avons utilisé la méthode préconisée par Lord (1959). Les yeux des sujets sont prélevés tout de suite après la mort et séjournent pendant un mois dans une solution de formol à $10 \%$. Les cristallins sont ensuite soigneusement disséqués et séchés $24 \mathrm{~h}$ dans une étuve à $100^{\circ} \mathrm{C}$. avant d'être pesés en ambiance sèche sur une balance monoplateau précise à $0,1 \mathrm{mg}$.

Tableau 1. Evolution du poids des cristallins $(\bar{x}$, moyenne exprimée en milligrammes; L.i., limite inférieure; L.s. limite supérieure) en fonction de l'âge (en mois) chez les Visons de couleur noire (1) et pastel (3). $V$, variance; $N$, taille de l'échantillon.

\begin{tabular}{|c|c|c|c|c|}
\hline àge & $q^{\prime}$ & L.i. $<\bar{x}<$ L.s. & $v$ & $N$ \\
\hline \multirow{4}{*}{6} & $q_{1}$ & $354<360<365$ & 404 & 47 \\
\cline { 2 - 5 } & $\sigma_{1}$ & $460<466<472$ & 732 & 87 \\
\cline { 2 - 5 } & $q_{3}$ & $371<383<396$ & 1279 & 31 \\
\cline { 2 - 5 } & $\sigma_{3}$ & $475<485<494$ & 851 & 36 \\
\hline \multirow{2}{*}{18} & $q_{1}$ & $434<442<450$ & 534 & 33 \\
\cline { 2 - 5 } & $\sigma_{1}$ & $555<567<578$ & 1578 & 43 \\
\hline 30 & $q_{1}$ & $436<448<460$ & 484 & 15 \\
\hline 42 & $q_{1}$ & $466<480<493$ & 331 & 9 \\
\hline 54 & $q_{1}$ & $473<494<514$ & 1058 & 12 \\
\hline 66 & $q_{1}$ & $494<513<532$ & 507 & 8 \\
\hline
\end{tabular}



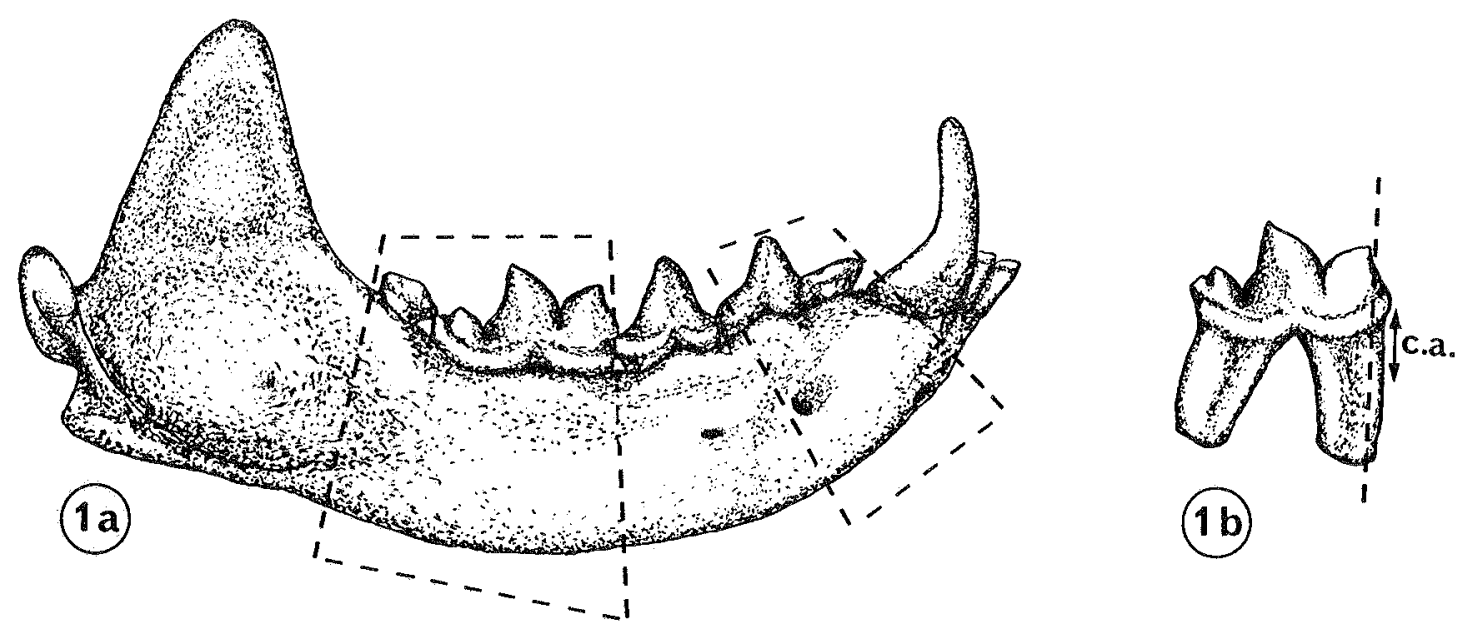

Fig. 1. (a) Régions de la mandibule utilisées pour l'étude des structures osseuses et dentaires. c.a., cément acellulaire. (b) Plans de coupe réalisés sur la carnassière (M1) de la mandibule.

\section{La squelettochronologie}

\section{Choix des animaux}

L'examen des pièces squelettiques a été réalisé sur un lot de 27 visons après sélection pour chaque classe d'âge de 3 à 6 animaux de robe et de sexe differrents.

\section{Choix des pièces squelettiques}

Enlow (1962) et de Ricqlès (1976) ont montré qu'en raison du remodelage squelettique, la croissance osseuse par apposition n'est jamais complètement achevée même chez les Mammifères, au moins localement. Dans un premier temps, il s'agit donc d'opérer un choix des pièces squelettiques supposées être le siège de paussées de croissance tout au long de la vie de l'animal. Cependant, la lecture sera d'autant plus aisée que cette croissance s'effectuera selon des modalités particulières. Il est souhaitable en effet qu'au sein du tissu choisi le dépôt osseux ne soit ni trop rapide, car alors le tissu n'enregistrera pas nettement les ralentissements ou les arrêts de l'ostéogénèse, ni trop lent, car le chevauchement des L.A.C. ne permettra pas une lecture correcte. Enfin, le remaniement osseux qui peut être à l'origine de l'élimination de plusieurs L.A.C. (Barbault et al. 1979; Castanet et Cheylan 1979), devra se présenter comme nul ou faible.

Les travaux consultés, en particulier ceux de Klevezal et Kleinenberg (1967), ainsi que notre expérience acquise sur le Chat haret des îles Kerguelen (Pascal et Castanet 1978), nous ont conduit à choisir dans un premier temps la mandibule, sans négliger pour autant l'examen d'un os long (l'un des fémurs).

L'étude du cément ne présente pas les mêmes difficultés que celle de l'os car il est rarement le siège de remaniements. Cependant, la faible épaisseur de ce tissu rend son observation assez délicate. C'est pour cette raison que l'essentiel des recherches a porté sur les dents pourvues d'une couche de cément particulièrement épaisse. C'est la démarche que nous avons suivie en sélectionnant la canine et la camassière (M1) de la mandibule.

Enfin, la dentine est observée en même temps que le cément sur les coupes de canines et de carnassières.

Choix des plans de coupe

Nous avons choisi de couper transversalement la mandibule depuis 1'arrière de la P3 jusqu'à l'arrière de la M2 (figure 1a). En opérant ainsi nous englobons la région osseuse qui a été étudiée par Klevezal et Kleinenberg (1967) chez le Vison. De plus, nous observons l'os en arrière de la M1 dans une région où il est peu soumis aux contraintes de pressions dues à la mastication qui, d'après certains auteurs (Enlow 1963; de Ricqlès 1976; inter alia), seraient à l'origine d'un fort remaniement. C'est d'ailleurs en arrière de la rangée dentaire que nous avons observé le plus faible taux de remaniement chez le Chat haret des îles Kerguelen (Pascal et Castanet 1978). Ce plan de coupe nous permet en outre de réaliser des coupes longitudinales de carnassières (figure $1 b$ ) qui restent dans la majorité des cas en connexion avec l'os mandibulaire. Nous observons donc simultanément l'os mandibulaire, le cément et la dentine de la carnassière.

La canine a été coupée transversalement depuis l'apex de la racine jusqu'au collet (figure $1 a$ ), ce qui est conforme au protocole utilisé par Ahnlund (1976). Malheureusement, les difficultés de montage n'ont pas toujours permis de garder en connexion les tissus osseux et dentaires.

Enfin, le fémur a été coupé transversalement au niveau de la diaphyse, région de l'os long où la corticale d'origine périostique est la plus épaisse et où, chez le Chat haret, nous avons trouvé la résorption la plus faible.

\section{Technique histologique}

Les pièces squelettiques (crâne complet et fémur) ont été prélevées immédiatement après le sacrifice, décharnées très grossièrement et plongées dans une solution de perborate de soude (approximativement $10 \mathrm{~g} / 100 \mathrm{~cm}^{3}$ ). Pendant $48 \mathrm{~h}$ les pièces ont été maintenues dans cette solution au sein d'une étuve thermostatée à la température de $80^{\circ} \mathrm{C}$. Pendant cette période, la solution a été renouvelée et une partie des chairs adhérentes éliminée à chaque changement de bain, jusqu'à l'obtention de pièces parfaitement propres. Cette méthode particulièrement rapide ne semble pas détériorer les parties minéralisées (en particulier le cément) et permet de conserver les dents en connexion (figures $4,5 a$ ).

La méthode de réalisation des préparations histologiques est inspirée directement de celle préconisée par Klevezal et Kleinenberg (1967). Nous l'avons rendue plus rapide (Pascal et 
Castanet 1978). Les coupes histologiques d'une épaisseur de 25 à $30 \mu \mathrm{m}$ sont colorées 30 à $45 \mathrm{~min}$ à l'hématoxyline d'Ehrlich, montées directement en milieu aqueux et observées à la loupe binoculaire ou au microscope (grossissement 8 à 400).

\section{Résultats}

Le poids des cristallins

Nous avons réuni l'ensemble de nos résultats sur le tableau 1. Le modèle linéaire qui rend compte de la croissance pondérale des cristallins en fonction de l'âge est de type semi logarithmique (Martinet 1966; Pépin 1973; Le Louarn 1971; Vincent et Quéré 1972). Dans un premier temps, nous avons appliqué cette transformation à l'ensemble de nos résultats portant sur les visons à la robe noire, séparant uniquement les mâles des femelles (figure 2). Nous avons obtenu les équations suivantes:

$$
\begin{aligned}
& \text { Femelles } \log t=0,016 P-3,989 \quad r=0,988 \\
& \text { Mâles } \quad \log t=0,012 P-3,721 \quad r=0,990
\end{aligned}
$$

où $t$ est l'âge en mois, $P$ le poids des cristallins en milligrammes et $r$ le coefficient de corrélation.

Le poids des cristallins augmente en fonction de l'âge quel que soit le sexe des animaux. Cependant, si cette évolution pondérale est rapide pendant la première année, elle se ralentit considérablement par la suite et ne permet plus de déterminer avec sécurité l'âge d'un animal de plus d'un an et demi.

Dans un deuxième temps, nous avons voulu savoir si la couleur de la robe avait une incidence quelconque sur les caractéristiques de la courbe âge/poids des cristallins. Le lot des animaux de 6 mois étant numériquement le plus important, c'est sur lui que nous avons testé cette hypothèse. Le poids des cristallins est, à âge égal, statistiquement différent selon que l'on a affaire à un lot de Visons de couleur pastel ou à un lot de Visons de couleur noire (tableau 1, figure 2). Les intervalles de confiance à 95\% ne se recouvrent pas et le test des moyennes $(t=$ $3,33>1,96$ ) permet d'affirmer qu'il y a, à âge égal, une différence hautement significative entre le poids des cristallins des Visons pastel et celui des cristallins des Visons noirs. Nous obtenons ici un résultat comparable à celui trouvé par Le Louarn (1971) sur le Campagnol des champs (Microtus arvalis Pallas). Sur le plan pratique une telle observation a des conséquences fâcheuses. En effet, chaque fois que l'on voudra étudier une population il sera indispensable, pour déterminer l'âge des individus sauvages, d'établir une courbe étalon à partir d'animaux d'âge connu en provenance de cette population. Ceci présente des difficultés parfois insurmontables, surtout quand l'élevage de l'espèce étudiée n'est pas réalisable.

\section{Les tissus squelettiques}

\section{Le fémur}

La cavité médullaire est importante et la surface dia-

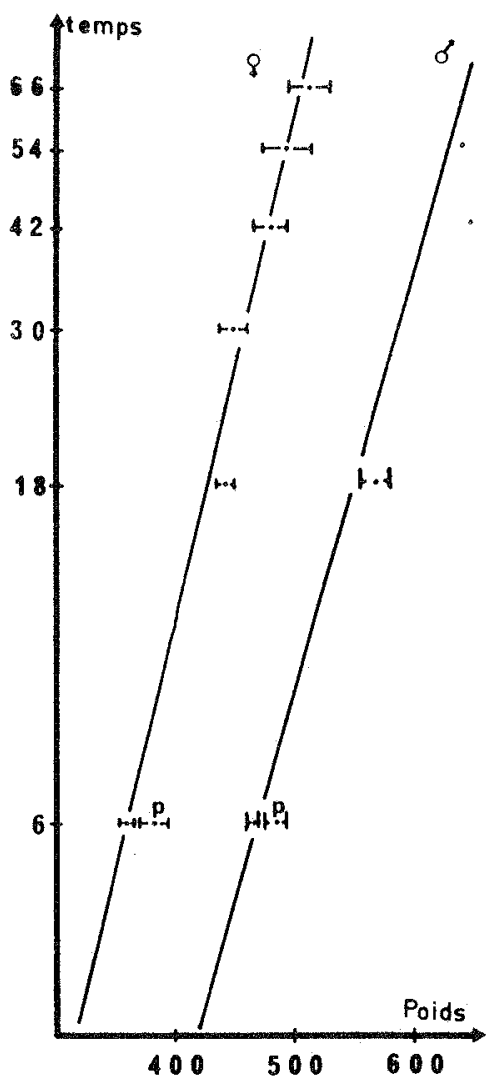

FIG. 2. Evolution des poids des cristallins ( $\mathrm{P}$ en milligrammes) en fonction de l'âge ( $t$ en mois) chez les mâles et les femelles de robe noire. Les valeurs obtenues pour la souche pastel $(p)$ sont figurées séparémment et identifiées par un $p$. Les données concernant les mâles de plus de 30 mois dont les effectifs sont trop faibles n'ont pas fait l'objet d'une analyse (leur présence est cependant notée), il n'en est pas fait cas dans le tableau 1.

physaire interne est tapissée d'une fine couche d'os lamellaire endostéal (figure $3 a$ ). Dans la corticale relativement épaisse une ligne de révẹrsion bien nette marque la frontière entre, d'une part, une couche interne d'os vascularisé, fortement remanié et, d'autre part, une mince couche périphérique d'os lamellaire peu vascularisé (figure $3 b$ ). Cet os lamellaire dont la croissance globale anisodiamétrique témoigne d'une dérive osseuse (Enlow 1963; de Ricqlès 1976) renferme des lignes hématoxylinophiles (figures $3 b$ ) dont le nombre n'est que rarement en corrélation avec l'âge des sujets. Les phénomènes de remodelage liés à la dérive spatiale de l'os sont fort probablement à l'origine de ce résultat en tout point comparable à celui que nous avons obtenu sur le Chat (Pascal et Castanet 1978). Nous avons donc abandonné l'étude de cette pièce squelettique.

\section{L'os mandibulaire}

L'observation au microscope optique de coupes transversales de mandibules d'animaux de 6 mois montre une 
cavité médullaire relativement importante tapissée d'un os endostéal lamellaire (figure 4). La corticale, épaisse, ne présente pas de lignes hématoxylinophiles et peut être subdivisée en deux parties. Une première couche, centrale, est constituée par un os fortement vascularisé, de type "à fibres entrecroisées," pourvu d'ostéoplastes globuleux et répartis au hasard. Ce tissu est le témoin d'une croissance rapide (Amprino 1949; Enlow 1966, 1969; de Ricqlès 1968, 1976, 1978). Progressivement, sans discontinuité (ligne de réversion ou ligne d'arrêt de croissance) cette structure laisse place à une faible épaisseur d'os de type "lamellaire," aisément identifiable au microscope polarisant. Cet os très peu vascularisé caractérise une croissance lente. A la périphérie de cette couche osseuse et dans la partie basale de la mandibule on note la présence de fibres de Sharpey.

La croissance pondérale chez les jeunes (figure 6) montre que le poids augmente rapidement jusqu'à l'âge de 5 mois et tout permet de supposer que c'est pendant ce laps de temps que se dépose la couche corticale d'os à fibres entrecroisées, témoin d'une ostéogénèse active. Passé cet âge, on note une stabilisation du poids suivie, à 6 mois, d'une décroissance sensible qui correspond à l'entrée de l'hiver. Le phénomène histologique qui caractériserait cette période est la mise en place de la fine couche d'os lamellaire. Il semble que nous ayons ici une bonne corrélation entre les modalités de l'ostéogénèse et des faits biologiques relatifs à la croissance des animaux.

Chez les animaux d'un an et plus, la corticale, épaisse, est constituée dans sa partie centrale par de l'os fortement vascularisé et remanié et, à sa périphérie, par une mince couche d'os lamellaire (figure $5 a$ ). La ligne hématoxylinophile la plus interne est toujours une ligne de réversion qui marque dans la plupart des cas la frontière entre l'os vascularisé remanié et l'os lamellaire. Cependant, chez certains sujets de 2 et 3 ans cette ligne est située dans l'os lamellaire. Dans ce cas, nous retrouvons le schéma typique décrit pour les animaux de 6 mois: un passage progressif et sans discontinuité de l'os fortement remanié à l'os lamellaire. La résorption qui est à l'origine de la ligne de réversion n'élimine donc pas toujours la couche d'os lamellaire déposée en continuité avec le cortex interne.
C'est à la base interne de la mandibule que l'os lamellaire périphérique est le plus épais. En plus de la ligne de réversion, cet os contient toujours une ou plusieurs lignes hématoxylinophiles en tous points comparables aux lignes d'arrêt de croissance décrites par Klevezal et Kleinenberg (1967). Cependant, on observe très souvent des dépôts lenticulaires d'os, ce qui permet de supposer que cette région est soumise à un remodelage séquentiel important. Nous avons dénombré ces L.A.C. sur une dizaine de préparations pour chaque animal et obtenu des résultats cohérents d'une préparation à l'autre (tableau 2). Le remaniement séquentie est probablement à l'origine de l'écart relativement important ( 2 ans dans certains cas) qui existe entre le nombre de L.A.C. et l'âge réel des animaux.

\section{La dentine}

Les lignes hématoxylinophiles ont tout d'abord été observées sur la dentine des coupes longitudinales de carnassières. La dentine palléale (Ørvig 1951; Bergot 1975) y est très homogène et nettement séparée de la dentine circum pulpaire par une première ligne sombre (figure $5 a$ ). Dans la dentine circum pulpaire, les lignes hématoxylinophiles sont plus ou moins "sombres." Le nombre total de ces lignes est toujours bien supérieur à l'âge de l'animal. Si l'on se limite aux lignes très sombres (figure $5 b$ ), ségrégation que l'on ne peut faire que subjectivement, 1 'âge est tout de même grandement surestimé (tableau 2). D'autre part, si l'on examine en détail la figure $5 b$, obtenue à partir d'une coupe longitudinale de carnassière d'un animal de 5 ans et demi, on peut dénombrer plus de huit lignes hématoxylinophiles dont quatre ou cinq peuvent être considérées comme sombres. Ce résultat suggérait que cet animal a effectivement 5 ans révolus. Cependant, l'épaisseur de la dentine comprise entre la cavité pulpaire et la première ligne sombre (chronologiquement la dernière mise en place) est considérablement plus importante que la couche de dentine déposée entre les autres lignes hématoxylinophiles. C'est un phénomène difficilement explicable si l'on suppose que ces lignes se mettent en place cycliquement et que la quantité de tissu déposé au cours de chaque cycle varie peu chez les animaux adultes.

Il n'y a donc aucune corrélation entre le nombre de

Abbréviations utilisés: Ce, cément; $\mathrm{Cm}$, cavité médullaire; $\mathrm{Cp}$, cavité pulpaire; De, dentine; Ds, desmodonte; LAC, ligne d'arrêt de croissance; Lc, ligne claire; $\mathrm{Lr}$, ligne de réversion; Ls, ligne sombre; Oe, os endostéal; Ol, os lamellaire.

FIG. 3. (a) Coupe transversale de la diaphyse fémorale d'une femelle de 3 ans. (b) Détail de la corticale d'une coupe transversale de la diaphyse fémorale d'une femelle de 3 ans.

Frg. 4. Coupe transversale de mandibule d'un vison mâle de 6 mois.

FIG. 5. (a) Coupe transversale de mandibule d'un vison mâle de 5 ans et demi. (b) Détail de la figure $5 a$ montrant les lignes sombres (flèches pleines) et les lignes claires que l'on eut observer dans la dentine au voisinage de la cavité pulpaire. (c) Détail de la figure $5 a$ montrant la région qui a été retenue pour la détermination de l'âge par le cément. La limite entre le cément et la dentine y est particulièrement visible et ne doit pas être confondue avec une L.A.C. (d) Détail de la figure $5 c$ montrant les L.A.C. dans le cément acellulaire. 


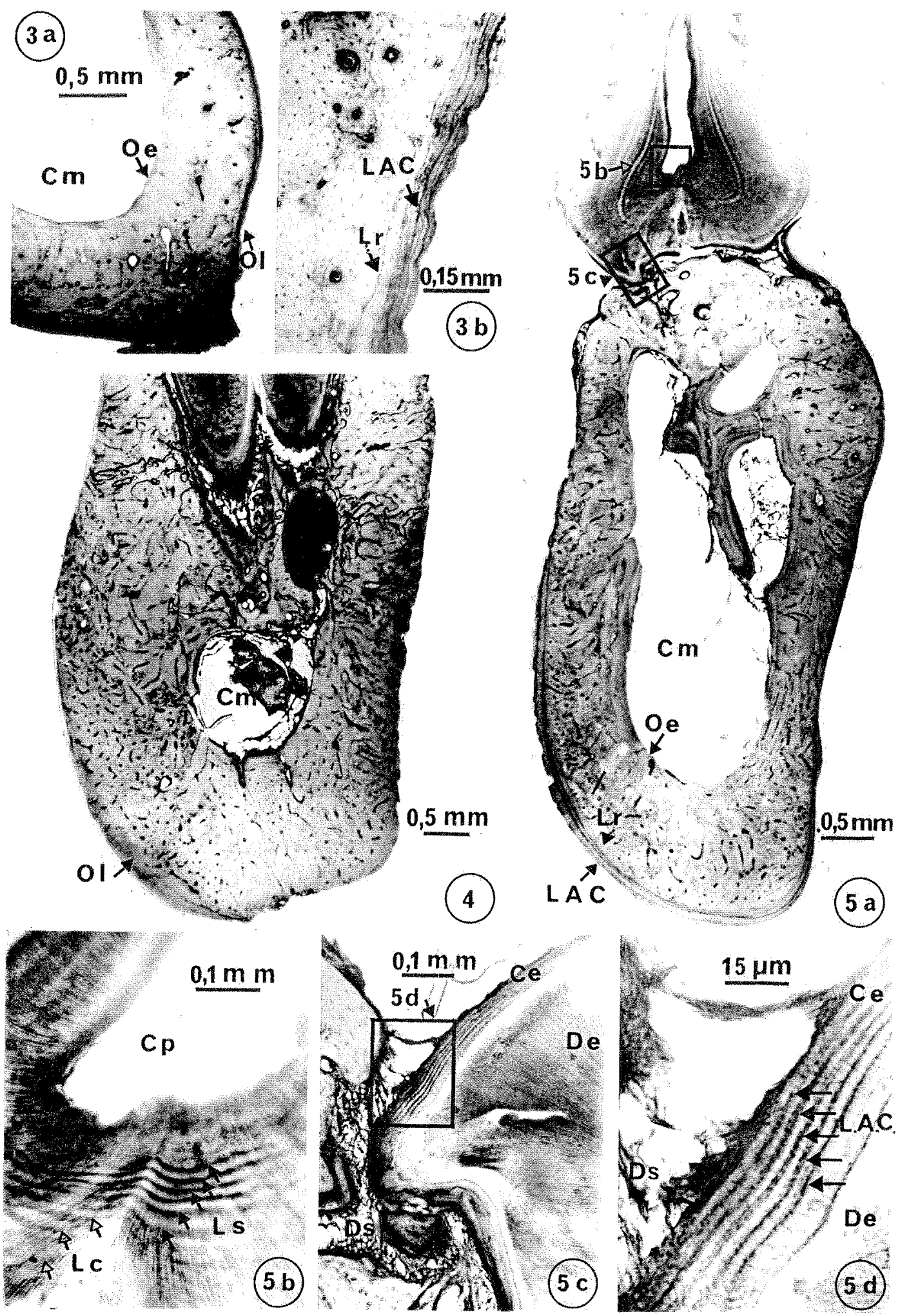




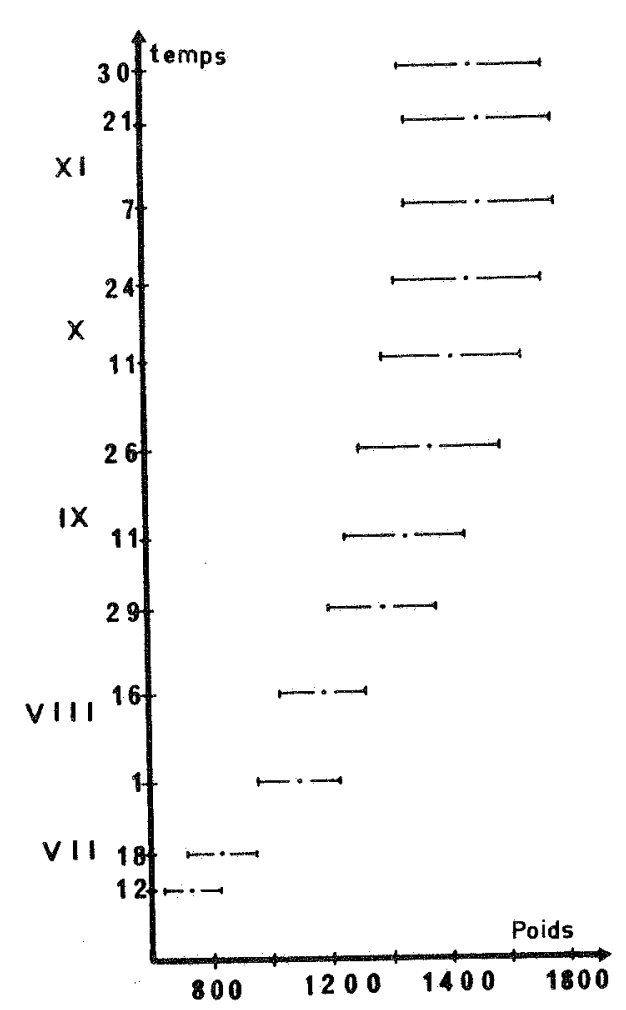

FIG. 6. Evolution pondérale ( $P$ en grammes) des animaux jeunes en fonction du temps ( $t$ en jours). On note à la fin du mois de novembre la décroissance du poids qui correspond à l'entrée de l'hiver.

L.A.C. observées dans la dentine et l'âge des sujets. pour confirmer cette conclusion, nous avons comparé chez trois animaux les résultats du dénombrement opéré sur la carnassière avec celui opéré sur la canine. Pour un même animal, non seulement les valeurs sont semblables mais les séquences des dépôts sont identiques. Cette observation est importante car elle indique que les marques de la dentine ne sont ni aléatoires ni soumises aux seules nécessités morphogénétiques particulières à chaque dent, mais qu'elles enregistrent bien un modèle de croissance individuel. Toutefois ce tissu renferme un tel nombre de marques qu'il enregistre manifestement autre chose qu'un cycle annuel. Chez cette espèce la dentine constitue donc un enregistreur trop sensible pour qu'il soit possible de l'utiliser pratiquement en squelettochronologie.

Une étude écologique, éthologique et physiologique très détaillée permettrait éventuellement de "décoder" les événements enregistrés dans la dentine, comme cela a été fait, par exemple, chez certains Pinnipèdes (Laws 1958; Carrick et Ingham 1962).

\section{Le cément}

Nous avons tout d'abord analysé le cément cellulaire des régions apicales et interradiculaires des racines de la
Tableau 2. Pour chacune des classes d'âge (en mois) est indiqué le nombre d'individus possédant $N$ lignes hématoxylinophiles dans le cément acellulaire, la dentine et l'os.

\begin{tabular}{|c|c|c|c|c|c|c|c|c|c|c|c|c|c|c|c|c|c|}
\hline & \multicolumn{6}{|c|}{ Cément } & \multicolumn{5}{|c|}{ Os } & \multicolumn{6}{|c|}{ Dentine } \\
\hline $\mathrm{ge}$ & 0 & 1 & 2 & & & 5 & 1 & 2 & 3 & 4 & 5 & 0 & 1 & 2 & 34 & 5 & $\infty$ \\
\hline 6 & 3 & & & & & $j$ & 3 & & & & & 3 & & & & & \\
\hline 18 & & 5 & & & & & 5 & & & & & 1 & 1 & 2 & & & 1 \\
\hline 30 & & 1 & 3 & & & & 1 & 3 & & & & & & 1 & & & 3 \\
\hline 42 & & & & 4 & & & & 1 & 1 & 2 & & & & & & 2 & 2 \\
\hline 54 & & & & & 5 & & & 1 & 1 & 2 & 1 & & & & & & 5 \\
\hline 66 & & & & & 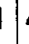 & 4 & & & 4 & 1 & 1 & & & & & & 5 \\
\hline
\end{tabular}

M1, en raison de son épaisseur importante. Ce cément présente chez certains individus des lignes hématoxylinophiles diffuses. Elles sont cependant rares et n'ont en général aucun rapport avec l'âge des animaux.

En revanche, le cément acellulaire, extrêmement mince, qui se dépose au voisinage du collet de la dent (figure $1 b$ ) présente des lignes hématoxylinophiles sombres très fines, bien discriminées et disposées régulièrement (figures $5 c, 5 d$ ). Sur certaines préparations, ces marques apparaissent plus nettes que sur d'autres. Ceci tient au fait que, si le plan de coupe est perpendiculaire à ces structures, elles n'apparaissent que faiblement contrastées et très serrées. Par contre, si le plan de coupe est légèrement oblique, l'épaisseur relative de ces structures augmente; elles deviennent ainsi beaucoup plus apparentes. Il semble aussi quie des coupes de 25 à 30 $\mu \mathrm{m}$ soient trop épaisses pour observer des structures aussi fines. En effet, l'épaisseur de cément entre deux L.A.C. est de l'ordre de $10 \mu \mathrm{m}$ (figure $5 d$ ). Des coupes de 15 à $20 \mu \mathrm{m}$ ou moins conviendraient certainement mieux. Il est important de conserver en connexion l'os mandibulaire et le cément sur les préparations car, dans le cas contraire, le contraste de lumière très important entre le cément et le milieu de montage ne permettrair pas de discerner les lignes hématoxylinophiles.

Le cément acellulaire des animaux de 6 mois ne contient aucune L.A.C. alors que chez les animaux plus agés on en observe toujours une ou plusieurs selon leur âge. La couche de cément qui s'est déposée la première est en général beaucoup plus épaisse que les suivantes (figure $5 d$ ) qui sont d'épaisseur à peu près constante. Quel que soit l'âge des animaux, il n'existe pas de ligne hématoxylinophile en périphérie de la dernière couche de cément. Comme tous nos animaux ont été sacrifiés à la même époque (courant décembre), il s'est donc écoulé un laps de temps voisin d'un cycle complet entre la mise en place de la dernière L.A.C. et la mort. 
Les lignes hématoxylinophiles ne se présentent pas toujours de façon aussi nette que sur la figure $5 d$. Souvent, sur un même préparation, des régions sont plus ou moins favorables à la lecture et, pour un même individu, certaines lames histologiques sont plus faciles à interpréter que d'autres. Nous avons donc recherché pour chaque spécimen la préparation et la région du collet la plus favorable (figure $1 b$ ), c'est-à-dire celle où le nombre de L.A.C. est maximum. C'est ce nombre qui à deux exceptions près, est en concordance avec l'âge réel des sujets (tableau 2). Dans ces deux cas, l'âge a été sousestimé d'un an et il semble que l'épaisseur des coupes histologiques soit responsable de cette erreur.

\section{Discussion}

L'os mandibulaire, la dentine, le cément, présentent tous trois des lignes hématoxylinophiles nettes. Cependant, ces trois tissus n'ont pas, au plan de la squelettochronologie, une valeur identique (tableau 2), Si la dentine se révèle comme un "enregistreur" très sensible, les lacunes de nos connaissances dans les domaines de l'écologie, de l'éthologie et de la physiologie de l'espèce ne nous permettent pas actuellement le "décodage" des évènements enregistrés par ce tissu et nous interdisent donc son utilisation dans le but pratique que nous poursuivons. Jusqu'à l'âge de 2 ans et demi, nous ne commettons pas d'erreur sensible dans la détermination de l'âge individuel en dénombrant le nombre de L.A.C. dans l'os mandibulaire. En effet, la ligne de réversion qui se met en place entre 6 mois et 1 an et demi ne perturbe pas l'évaluation de l'âge par élimination de la première L.A.C. En outre, les phénomènes de dérive osseuse n'apparaissent de façon notable qu'après l'âge de 2 ans et demi. Cependant, à partir de cet âge l'os mandibulaire ne constitue plus un indicateur suffisamment fiable pour être utilisé. Par contre, le cément acellulaire du voisinage du collet de la carnassière a permis de déterminer en toute sécurité l'âge individuel sur l'ensemble des classes examinées.

La présence de lignes hématoxylinophiles dans les trois tissus étudiés pose le problème de la synchronisation de leur dépôt. Il est relativement facile de montrer que, chez un même individu, les lignes hématoxylinophiles observées dans la dentine de deux dents différentes sont déposées vraisemblablement de façon synchrone. En effet, l'espacement entre les différentes lignes, leur coloration plus ou moins marquée, leur épaisseur relative sont autant d'indices qui permettent d'aboutir à cette conclusion avec quelques raisons. En revanche, il serait abusif d'étendre un pareil procédé à la comparaison des marques contenues dans les trois types de tissus étudiés. Aucun repère temporel ne permet d'affirmer que les lignes hématoxylinophiles observées dans ces différents tissus se sont déposées au même moment.

La mise en place des L.A.C. dans le cément se fait très certainement après le mois de décembre chez les Visons de France. En effet, d'une part, les animaux de 6 mois n'ont pas de L.A.C., que ce soit dans le cément ou dans l'os. D'autre part, une couche de cément d'épaisseur analogue à celle qui s'est déposée au cours des cycles précédents est mise en place après la dernière L.A.C. chez les individus plus âgés (figure $5 d$ ); enfin, le cément des animaux de plus de 6 mois ne présente pas de L.A.C. à sa périphérie. Il est donc fort probable que les L.A.C. se forment pendant l'hiver et que la reprise de croissance des tissus calcifiés se fait en même temps que la reprise du poids, donc au printemps.

Nous n'avons pas trouvé, comme Klevezal et Kleinenberg (1967), une bonne corrélation entre le nombre de L.A.C. dans l'os mandibulaire et l'âge des animaux. Klevezal (1973), citée par Rudge (1976), apporte peutêtre une explication à cette divergence en précisant que les lignes de croissance annuelles se déposent d'une façon plus nette et régulière chez les animaux soumis à un climat continental aux saisons plus tranchées que chez ceux soumis à un climat océanique. Les poussées de croissance des tissus calcifiés répondraient donc à un rythme endogène modulé et (ou) synchronisé par des facteurs exogènes (Castanet et Naulleau 1974; Castanet et al. 1977; Meunier et al. 1979; Meunier et Pascal 1980). Notons que dans ce cas précis les variations des disponibilités alimentaires ne sont pas directement responsables de l'enregistrement des cycles annuels dans les tissus calcifiés puisque les animaux ont été élevés sans pénurie hivernale de nourriture.

Nous avons vu que chez le Vison le poids des cristallins constitue un excellent critère d'âge jusqu'à 1 an. Si l'on peut établir pour la population étudiée la courbe des poids des cristallins en fonction de l'âge, cette technique est alors extrêmement rapide et fiable dans son domaine de validité. Les jeunes individus constituant généralement une classe numériquement très importante dans les populations sauvages, l'intérêt pratique de cette méthode est donc évident.

Dans le cadre de l'étude de la structure et de la dynamique d'une population de Mustélidés sauvages, nous proposons donc, sur un plan purement pratique, de conserver systématiquement le crâne et les yeux des spécimens prélevés et d'opérer rapidement la partition de l'échantillon en jeunes de l'année et en adultes sur la base du poids des cristallins et de la chronologie des éruptions dentaires. Les individus plus âgés sont alors répartis en classes d'âge annuelles grâce à l'utilisation de la squelettochronologie appliquée conjointement au tissu osseux mandibulaire et au cément acellulaire de la carnassière.

Enfin, en l'absence d'un lot de référence d'animaux d'âge connu, il demeure possible de se servir de l'étude détaillée des structures osseuses pour déterminer l'âge individuel. Il est alors indispensable d'interpréter cor- 
rectement la signification fonctionnelle de ces structures et, pour cela, de réunir une somme importante d'informations diverses portant sur la biologie, l'écologie, la physiologie etc. de l'espèce étudiée. Les résultats de la squelettochronologie sont alors validés par la cohérence et la convergence d'un faisceau d'informations. Il s'agit alors d'utiliser la notion de "présomptions concordantes" (circonstancial evidences), énoncée par Peabody (1961), comme nous l'avons fait à propos du Chat haret des îles Kerguelen (Pascal et Castanet 1978).

La squelettochronologie n'est donc pas une panacée, ni une technique de routine capable de donner dans l'absolu des classe d'âge fiables par lecture directe et non critique. Ses résultats sont d'autant plus fiables et significatifs qu'elle est menée de front avec une étude biologique exhaustive de la population étudiée.

\section{Remerciements}

Nous tenons à remercier ici M. Rougeot et M. Allain, Laboratoire des Pelages, Toisons et Fourrures, Centre National de la Recherche Zootechnique 78350 Jouy en Josas, France, qui nous ont permis d'exécuter les prélèvements nécessaires à cette étude et qui nous ont aimablement communiqué les renseignements relatifs à la conduite de l'élevage et à la croissance pondérale des animaux.

AHNLUND, M. 1976. Age determination in European badger, Meles meles L. Z. Saeugetierkd. 41: 119-125.

Amprino, R. 1947. La structure du tissu osseux envisagée comme expression de différences dans la vitesse de l'accroissement. Arch. Biol. (Liège), 58: 315-330.

Aulerich, R. J., et D. R. SCHWINDLER. 1968. The dentition of the Mink (Mustela vison). J. Mammal. 49: 488-494.

Barbault, R., J. Castanet, H. Francillon et A. de RicQLès. 1979. Détermination de l'âge chez un Anoure déserticole Bufo pentoni Anckson 1893. Terre Vie, 33: 129-141.

Bergor, C. 1975. Mise en évidence d'un type particulier de dentine chez la Truite (Salmo sp.; Salmonidé, Téléostéens). Bull. Soc. Zool. Fr. 100: 587-594.

Birney, E. C., et E. D. Fleharty. 1966. Age and sex comparisons of wild mink. Trans. Kansas Acad. Sci. 69: 139-145.

Bourliere, F., et F. Sprtz. 1975. Les critères d'âge chez les Mammifères. Dans Problèmes d'écologie. La démographie des populations de Vertébrés. Masson, Paris. pp. 53-75.

Carrick, R., et S. E. Ingham. 1962. Studies on the southern elephant seal Mirunga leonina L. II. Canine tooth structure in relation to function and age determination. CSIRO Wildl. Res. 7(2): 102-118.

CASTANET, J. 1974. Etude histologique des marques squelettiques de croissance chez Vipera aspis (L.) (Ophidia, Viperidae). Zool. Scr. 13: 137-151

Castanet, J., et M. Cheylan. 1979. Les marques de croissance des os et des écailles comme indicateur de l'âge chez Testudo hermanni et Testudo graeca (Reptilia, Chelonia, Testudinidae). Can. J. Zool. 57: 1649-1665.
Castanet, J., F. J. Meunier et A. de Ricqlès. 1977. L'enregistrement de la croissance cyclique par le tissu osseux chez les Vertébrés poikilothermes: données comparatives et essai de synthèse. Bull. Biol. Fr. Belg. 111: 183-202.

Castanet, J., et G. Naulleau. 1974. Données expérimentales sur la valeur des marques squelettiques comme indicateur d'âge chez Vipera aspis (L.) (Ophidia, Viperidae). Zool. Scr. 3: 201-208.

Craighead, J. J. 1970. Age determination of Grizzly bears from fourth premolar tooth sections. J. Wildl. Manage. 34: 353-363.

ELDER, W. H. 1951. The baculum as an age criterion in mink. J. Mammal. 32: 45-50.

ENLow, D. H. 1962. A study of the post natal growth and remodeling of bone. Am. J. Anat. 110: 79-102.

1963. Principles of bone remodeling. Charles C. Thomas, Springfield.

1966. An evaluation of the use of bone histology in forensic medicine and anthropology. Dans Studies on the anatomy and function of bone and joints. Editeur: F. G. Evans. Springer Verlag, Berlin. pp. 93-112.

1969. The bone of reptiles. Vol. 1. Dans Biology of the Reptilia. Editeur: C. Gans. Academic Press, New York. pp. $45-80$.

Grue, H., et B. Jensen. 1973. Annular structures in canine tooth cementum in red Foxes (Vulpes vulpes L.) of known age. Dan. Rev. Game Biol. 8: 1-12.

HiBbARD, E. A. 1956. Age ratios in wild mink populations, J. Mammal. 38: 412-413.

JENSEN, B., et L. B. NIELSEN. 1968. Age determination in the red fox (Vulpes vulpes $\mathrm{L}$.) from canine tooth sections. Dan. Rev. Game Biol. 5: 1-15.

JONSGARD, A. 1969. Age determination of marine mammals. Dans The biology of marine mammals. Editeur: $\mathrm{H}$. Andersen. Academic Press, New York.

KLevezal, G. A. 1973. Some limitations and new possibilities of using layers in tooth and bone tissue for age determination in mammals. Zool. Zh. 52: 757-765.

Klevezal, G. A., et S. E. KleinenberG. 1967. Age determination of mammals from annual layers in teeth and bones. Traduit du Russe par Israël Program for Scientific Translations, Jerusalem, 1969.

Laws, R. M. 1958. A new method of age determination in mammals with special references to the elephant seal (Mirunga leonina). Falkland Islands Depend. Surv. Sci. Rep. 2: 1-11.

LECHLEITNER, R. R. 1954. Age criteria in mink, Mustela vison. J. Mammal. 35: 496-503.

Le Louarn, H . 1971. Détermination de l'âge par la pesée des cristallins chez quelques espèces de rongeurs. Mammalia, 35: 636-643.

LoRD, R. D. 1959. The lens as an indicator of age in cottontail rabbits. J. Wildl. Manage. 23: 358-360.

Martinet, L. 1966. Détermination de l'âge chez le Campagnol des champs (Microtus arvalis) par la pesée du cristallin. Mammalia, 30: 425-430.

Meunier, F. J., M. Pascal et G. Loubens. 1979. Comparaison de méthodes squelettochronologiques et considérations fonctionnelles sur le tissu osseux acellulaire d'un 
Osteichthyen du lagon Néo-Caledonien, Lethrinus nebulosus (Forskal, 1775). Aquaculture, 17: 137-157.

Meunier, F, J., et M. Pascal. 1980. Quelques données comparatives sur la croissance périodique des tissus squelettiques chez les Osteichthyens. Bull. Soc. Zool. Fr. 105: 337-342.

MoRRIs, P. A. 1970. A method for determining absolute age in the hedgehog. J. Zool. London, 161: 277-281.

$\emptyset_{\mathrm{RVIG}}, \mathrm{T}, 1951$, Histologic studies of placoderm and fossil elasmobranch. I. The endoskeleton with remarks on the hard tissues of lower vertebrates in general. Ark. Zool. 2: $321-454$.

Pascal, M., et J. Castranet. 1978. Méthodes de détermination de l'âge chez le Chat haret des îles Kerguelen. Terre Vie, 32: 529-555.

PAUL, J. R. 1968. Baculum development in mink. Trans. Illinois Acad. Sci. 61(3): 308-309.

PEABODY, F. E. 1961. Annual growth zones in vertebrates (living and fossils) J. Morphol. 100: 11-62.

PÉPIN, D. 1973. Recherche d'un critère d'âge chez le Lièvre, Lepus europaeus. Ann. Zool. Ecol. Anim. 5: 271-281.

RICQLÈs, A. DE. 1968. Recherches paléohistologiques sur les os longs des tétrapodes. I. Origine du tissu osseux plexiforme des Dinosauriens Sauropodes. Ann. Paleontol. Vertebr. 54: 133-145.

1976. Recherches paléohistologiques sur les os longs des tétrapodes. VII. 2ème part. Sur la classification, la signification fonctionnelle et l'histoire des tissus osseux des tétrapodes. Ann. Paleontol. Vertebr. 62: 71-126.

1978. Recherches paléohistologiques sur les os longs des tétrapodes. VII. Sur la classification, la signification fonctionnelle et l'histoire des tissus osseux des tétrapodes. Ann. Paleontol. Vertebr. 64: 153-184.

RUDGE, M. R. 1976. Ageing domestic sheep (Ovis aries L.) from growth lines in the cementum of the first incisor. N.Z. J. Zool. 3: 421-424.

SERGEANT, D. E. 1967. Age determination of land mammals. Z. Saeugetierkd. 32: 297-300.

Stephenson, A. B. 1977. Age determination and morpholo. gical variation of Ontario otters. Can. J. Zool. 55: 1578.

Stonberg, R. P., et C. J. Jonkel. 1966. Age determination of black bears by cementum layers. J. Wildl. Manage. 30: $411-414$.

Van Soest, R. W., et P.J. H. VAN Bree. 1970. Sex and age composition of a stoat population (Mustela erminea Linnaeus, 1758) from a coastal dune region of the Netherlands. Beaufortia Ser. Misc. Publ. Zool. Mus. Univ. Amsterdam, 226: 51-77.

VINCENT, J. P., et J. P. Quéré. 1972. Quelques données sur la reproduction et sur la dynamique des populations du Rat musqué Ondatra zibethica L. dans le nord de la France. J. Zool. Ecol. Anim. 4: 395-415. 